\title{
EXPERIENCES WITH FECAL MICROBIOTA TRANSPLANTATION IN CLOSTRIDIUM DIFFICILE INFECTIONS VIA UPPER GASTROINTESTINAL TRACT
}

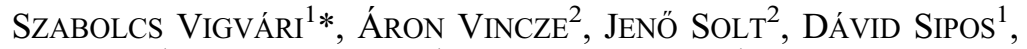 \\ ZSÓFIA FEISZT ${ }^{1}$, BEÁtA KOVÁCs ${ }^{1}$, ÁGNes KAPPÉTER ${ }^{1}$ and ZOLTÁN PÉTERFI ${ }^{1}$ \\ ${ }^{1}$ Department of Infectology, 1st Department of Internal Medicine, \\ University of Pécs, Pécs, Hungary \\ ${ }^{2}$ Department of Gastroenterology, 1st Department of Internal Medicine, \\ University of Pécs, Pécs, Hungary
}

(Received: 2 October 2018; accepted: 21 November 2018)

Dramatic changes in the epidemiology of Clostridium difficile infections have been reported from the western world in the past decade. The proportion of severe cases is significantly elevating and clinicians now have to contend with the problem of additional and more frequent episodes of recurrences including an upward trend in the mortality rate. This situation led us to investigate the possibility of the fecal microbiota transplantation (FMT). An amount of $100 \mathrm{ml}$ of fecal microbiota solution was instilled into a nasojejunal (NJ) tube in 16 cases and into a nasogastric (NG) tube in 44 cases. In all of the cases, where the solution was instilled via nasojejunal tubes, the symptoms resolved within $24 \mathrm{~h}$. We did not note any recurrences in this group. When the material was flushed in through nasogastric tubes, the symptoms resolved in $39(88.64 \%)$ cases within $24 \mathrm{~h}$. In this group, we have experienced a recurrent episode of $C$. difficile infection in five $(11.36 \%)$ cases. Three of them were cured with a second transplantation. We have found that in our practice the upper gastrointestinal tract methods had the primary cure rate of $91.67 \%$, whereas the secondary cure rate is $96.67 \%$. When we compared the NJ and NG methods, we have found that the differences in the outcomes are not significant statistically ( $p=0.3113$ using Fisher's exact probability test). In conclusion, FMT proved to be very effective, particularly in recurrent infections and in cases where conventional treatment had failed.

Keywords: fecal microbiota transplantation, nasogastric tube, nasojejunal tube

*Corresponding author; E-mail: szabolcs.vigvari@gmail.com 


\section{Introduction}

Clostridium difficile, a Gram-positive, obligate anaerobic, spore-forming bacillus, was first isolated from the feces of healthy neonates in 1935 [1]. It was initially believed to be non-pathogenic, but in 1978, C. difficile was identified as the leading cause of antibiotic-associated diarrhea and a major cause of pseudomembranous colitis [2]. The epidemiology of $C$. difficile infection (CDI) has recently undergone a dramatic change worldwide. Between 1996 and 2003, the prevalence of CDI in the USA doubled, reaching 61 per 100,000 [3], and the rate of CDI listed as any diagnosis of hospitalized patients rose from 3.82 per 1,000 discharges in 2000 to 8.75 per 1,000 discharges in 2008 [4]. Simultaneously, greater numbers of severe and lethal cases or treatment failures were reported from Quebec (Canada) and in the USA [5]. In Pécs, a city in southwest Hungary, the incidence and mortality (12\%) of CDI have increased since 2008. Considerable effort has been devoted to the search for new strategies of recurrent CDI. It was recently suggested that fecal microbiota transplantation (FMT) is a relatively safe, low cost, and effective solution of CDI that fails to resolve in response to traditional antibiotic regimens, especially in recurrences [6]. Although the first transplantation in humans was reported in 1958 [7], the idea of the transplantation of viable gastrointestinal (GI) microbes from one individual to another is much older. It was probably the Italian anatomist Fabricius Aquapedente who first described this method in animals, as "transfaunation" [8].

\section{Materials and Methods}

\section{Patient indications}

However, there is no clear evidence as to the exact indications of FMT, but the recently published international literature strongly indicates that it is an effective option in recurrent CDI, primarily for those cases where conservative therapeutic solutions have failed to eliminate the infection $[6,9,10]$. Study participants were recruited based on criteria of FMT, published in consensus guidelines [11], and adapted to local circumstances. Subjects with refractory or recurrent active CDI were included. Major exclusion criteria included acute abdomen, signs of perforation, recent operation with the opening of the upper or lower GI tract, upper GI tract hemorrhage, signs of ileus, and uncontrollable vomiting. 
Proper donor screening is essential to avoid the transmission of communicable diseases. Each donor served as a volunteer and was initially screened by means of a questionnaire to exclude potentially high-risk individuals (e.g., drug use, unprotected sexual behavior or a history of extensive travel, illness, or antibiotic intake in the past 6 months). Donated feces were screened for parasites, entero-pathogenic bacteria, and $C$. difficile, whereas the serum was screened for HIV-1 and HIV-2, EBV, CMV, hepatitis A, B, and C, and Treponema pallidum.

\section{Preparation of the sample}

Neither the exact amount of donated feces needed for the transplantation nor the volume of fluid has been clearly determined. Meta-analyses suggest that a larger volume of feces will increase the success rate and reduce the relapse rate, since ideal clinical results seem to be greatest (97\%) when more than $500 \mathrm{ml}$ of suspension is transferred (while the resolution rate was found $80 \%$ when less than $200 \mathrm{ml}$ is flushed in), and four times higher relapse rates have been reported when less than $50 \mathrm{~g}$ of stool is donated $[6,8]$. Most authors recommend the use of normal saline, but some have used milk in view of the finding in which patients with recurrent CDI excrete lower amounts of short-chain fatty acids into their feces as compared to healthy controls $[12,13]$.

We used freshly prepared transplant material, originated from a relative or a household member of the patients in each of the cases. The donated feces were collected and transported to our department on the day of the planned FMT. A 60-g sample was homogenized in a mortar and suspended in $200 \mathrm{ml}$ of normal saline $(0.9 \%)$. The suspension was then filtered through $4 \times 4 \mathrm{~cm}$ sheets of sterile gauze into another container, and $100 \mathrm{ml}$ of the filtrate was absorbed into a syringe. Entire steps of the preparation were performed in a laminated flow box, and sterile devices were employed. Within $6 \mathrm{~h}$ following the collection of the feces by the donor, the solution was infused into the patient.

\section{Recipient preparation}

In our department, when the condition of the patient permitted, antibiotic administration was halted 4 days prior to the procedure. In serious cases, this period was reduced to 1 day. In each case, thorough purging was applied on the day prior to the intervention, in order to increase the success of the procedure, by 
flushing away residual feces, antibiotics, toxins, $C$. difficile bacteria, and spores to the maximum attainable extent. The patients consumed nothing from midnight, and in the morning before the procedure, they received a double dose of proton pump inhibitor (PPI) intravenously. This increases the chances of survival of the transplanted flora by reducing the gastric acid output. One hour prior to the transplantation, $20 \mathrm{mg}$ of metoclopramide was administered intravenously, to prevent vomiting and to facilitate bowel movement in order to promote the delivery of the transplantation material to the colon.

\section{FMT delivery}

The first reported FMT was achieved via a retention enema [7]. Since then, three main types of FMT delivery have been developed. Clinicians tend to prefer lower GI tract delivery, which can be performed in a highly effective way $[14,15]$. Upper GI tract delivery methods also seem to be effective and time efficient according to some studies [16-18]. Each technique has its advantages and disadvantages and in the end, the choice of the optimal procedure should be a patient-centered decision, based on both the wishes of the patient and the clinical aspects, e.g., the patient's general condition, the available equipment at site, and skills and previous experiences of the medical staff.

Recently published results led us to use upper GI delivery methods, including nasoduodenal (ND) and nasogastric (NG) tubes [19, 20]. Before the NG tube was inserted, the distance was measured from the tip of the patient's nose, loop around their ear, and then down $5 \mathrm{~cm}$ below the xiphoid process. The tube was then marked at this level to ensure that the tube has been inserted far enough into the patient's stomach. The end of the plastic tube was lubricated (anesthetic spray have been applied before the insertion) and inserted into one of the patient's anterior nares. The tube was directed straight toward the back of the patient as it moves through the nasal cavity and down into the throat. When the tube enters the oropharynx, the patients were asked to mimic swallowing or some amount of water was given to them to sip through a straw, and the tube continues to be inserted as the patient swallows. To verify proper placement, air was injected into the tube; if it was detected in the stomach by auscultation, the tube was declared in the correct position.

The NJ feeding tube was placed by one skilled doctor. Some gastroenterologists were also involved in the fluoroscopy. Additional sedation was not required for fluoroscopic tube placement. A portable C-arm fluoroscope was positioned over the supine patient's abdomen. The timing of the procedure began when the feeding tube entered the nares. When the tube was advanced to 
50-55 cm, its position was evaluated by intermittent fluoroscopy. The operators rotated the feeding tube to facilitate passage to the pylorus. Fluoroscopy was used intermittently or continuously as needed. When the tip of the feeding tube was beyond the pylorus, it was gently advanced as far as possible. Placement of the tube beyond the third portion of the duodenum was preferred. Finally, a fluoroscopic print was obtained after $10-15 \mathrm{ml}$ of meglumine diatrizoate had been injected into the feeding tube.

\section{Cure rate}

We defined the primary cure rate as the percentage of cases in which the symptoms resolved without recurrence within 6 weeks after the FMT, and the secondary cure rate as the percentage of cases in which the symptoms resolved after a second FMT [14]. IDSA/SHEA guidelines do NOT recommend $C$. difficile testing in patients who do not have symptoms, because patients can be colonized with $C$. difficile and not develop disease [11,21].

\section{Results}

\section{Demographic data}

We performed FMT on a total of 60 patients, of whom 37 (61.67\%) were females. The mean age was 71.48 years, including a range from 26 to 88 years of age. The average number of recurrent infections was 2.24 (range: $0-8$ ). In 40 cases $(66.67 \%)$, the patients exhibited a severe infection during the transplantation (Table I).

\section{Clinical outcomes}

In all 16 initial cases, where the solution was instilled through ND tubes, we noted that diarrhea had stopped within $24 \mathrm{~h}$, as did all of the other symptoms. No recurrences occurred in this group. Following 1 week, it should be noted that diarrhea resumed in the case of a 70-year-old female who previously had eight microbiologically proven recurrences of CDI. Clostridial toxins could not be detected and the bacterium could not be cultured from her stools after the FMT. Detailed gastroenterological examinations subsequently revealed a late onset of irritable bowel syndrome in this patient. 
Table I. Demographic and clinical characteristics of the patients

\begin{tabular}{lcc}
\hline & $\begin{array}{c}\text { FMT via ND sonde } \\
(N=16)\end{array}$ & $\begin{array}{c}\text { FMT via NG sonde } \\
(N=44)\end{array}$ \\
Characteristics & $67.8(35-88)$ & $76.7(51-89)$ \\
Median age (years; range) & $9(56.2)$ & $28(63.64)$ \\
Number of females & $2.7(1-8)$ & $1.75(0-3)$ \\
Median number of recurrences of CDI (range) & $11(68.75)$ & $30(68.18)$ \\
Number of previous failure of vancomycin therapy (\%) & $16(100)$ & $37(84.09)$ \\
Number of previous antibiotic use (\%) & $11(68.75)$ & $26(59.09)$ \\
Number of use of proton pump inhibitor (\%) & $7.2(5-15)$ & $5.2(5-10)$ \\
Median stool frequency/24 h (range) & 7,900 & 12,000 \\
Median leukocyte count/mm (range) & $(2,900-27,000)$ & $(5,600-19,700)$ \\
& $80.75(45-173)$ & $109.12(37-270)$ \\
\hline
\end{tabular}

Note: FMT: fecal microbiota transplantation; CDI: Clostridium difficile infection; ND: nasoduodenal; NG: nasogastric.

When the inoculum was flushed in through NG tube, all the patients improved within 48 h. In $88.64 \%$ (39) of these cases, we did not experience any recurrences during 6 weeks; however, in five cases (11.36\%), microbiologically confirmed CDI recurred. These patients were aged (over 75 years), with a severe underlying condition, and it is important to note that one of them had previously been given antibiotics to treat his urinary tract infection caused by multidrug-resistant organisms. After the second transplantation, three of the five patients were cured, and thus the secondary cure rate in this subgroup has been found to $95.45 \%$. In our hands, the overall primary cure rate of upper GI tract FMT was $91.67 \%$, whereas the overall secondary cure rate was $96.67 \%$.

It is important to note the recurrence rate appeared to be lower when the solution was instilled through ND tubes. Although the difference $(p=0.3113$, using Fisher's exact probability test) between the two groups was statistically not significant, we consider it to be clinically important.

Nausea was observed in one case, but could be controlled with vitamin B6 and metoclopramid. No other side-effects or reactions were detected, and therefore we cannot support the view in which the "yuck" factor is a relevant obstacle.

\section{Discussion}

Treating CDI and mainly recurrent CDI are the therapeutic challenges for patients and physicians. Current treatment options include repeated or prolonged courses of antibiotics with limited success rates, and there is lack of alternative therapeutic options (immunoglobulin preparations from vaccinated cows and probiotics). Participants of this study were recruited from the patients referred 
to our ward between April 2013 and September 2015. All of them suffered from refractory or recurrent episode of CDI. Indications and contraindication were based on the recommendations of consensus guidelines and were adapted to local circumstances [22].

One of the modes of performing FMT available today is upper GI tract administration including NG tubes, duodenal tubes, and endoscopy/gastroscopy. According to the recorded data, upper GI tract FMT requires smaller volumes of fecal suspensions, and it also has a high cure rate $[20,21]$. We prefer to use NG tubes in our practice, because it has the advantage that it requires less patient preparation, clinical time, and less patient inconvenience and cost than other routes of administration. This method does not require the specialized skills of endoscopic procedures and it can be performed at the patients' bedside. Each patient was given a double dose of PPI intravenously at least $2 \mathrm{~h}$ prior to the procedure. This increases the chances of survival of the transplanted flora by reducing the gastric acid output. One hour prior to the transplantation, $20 \mathrm{mg}$ of metoclopramide was administered intravenously, to prevent vomiting and to facilitate bowel movement in order to promote the delivery of the transplantation material to the colon. However, in order to maximize the success rate, we decided to perform FMT via ND route in those 16 cases $(26.67 \%)$, where patients still complained of symptoms of gastroesophageal reflux disease, despite taking double dose of PPI regularly.

We used freshly prepared samples from healthy adults who were household members or close relatives of the patients in each of the correlating case. Unfortunately, a lot of patients did not have a relative, who could have been suitable as a donor.

All our patients were hospitalized and discharged 3 or more days after FMT. Possible adverse events were provoked by the medical staff of our ward based on a self-report of the patients. Fever, GI symptoms, headache, fatigue, and rash were the main symptoms evaluated. No serious adverse events or recurrences were observed. No patient vomited within $24 \mathrm{~h}$ of administration. Mild adverse events, e.g., bloating, abdominal cramp, and nausea were reported in just one case (1.67\%).

In our hands, the overall primary cure rate of upper GI tract FMT was $91.67 \%$, whereas the overall secondary cure rate was $96.67 \%$.

Although we experienced an excellent clinical outcome, it is important to note that this study has major limitations. The design of a multicentrical, randomized study involving larger number of patients and with active comparators should be considered in the future. The other major limitation is the problem of long-term follow-up. All study participants were contacted through phone 6 weeks and 3 months after the FMT. They were asked to complete a short questionnaire concerning on their bowel movement, abdominal pain, and general well-being, 
recurrent episode of CDI, antibiotic intake since FMT, and new medical conditions occurring since FMT. No new medical conditions were reported. However, it is important to note that these answers were not confirmed by a healthcare professional (e.g., general practitioner or a nurse), except those (17 patients; $28.33 \%$ ) who were inhabitants of a healthcare facility for elderly. No control stool tests were performed. In order to evaluate the long-term efficacy (6 or 12 months), additional interviews will be required.

\section{Conclusions}

FMT via upper GI tract has a high cure rate nearing 100\%, and it is reported safe that supports the viability of its use. We have to note, due to a lack of clinical trials, it is only offered to patients with two or more relapses, and it remains as a last rescue therapy. It is clear that further investigations are required to determine its place in our therapeutic arsenal and the best protocol to perform FMT.

\section{Acknowledgements}

All FMT procedures were carried out with the written ethical approval (permission number: 16014) of the appropriate Scientific and Ethics committee (Egészségügyi Tudományos Tanács Tudományos és Kutatásetikai Bizottság, ETT-TUKEB), and the written informed consent of the patients was obtained for participating in this study.

\section{Conflict of Interest}

The authors work in a University Hospital, where fecal microbiota transplantation is a treatment option for patients suffering from recurrent or severe CDI.

\section{References}

1. Hall, I. C., O’Toole, E.: Intestinal flora in newborn infants: With a description of a new pathogenic anaerobe, Bacillus difficile. Am J Dis Child 49, 390-402 (1935).

2. Larson, H. E., Price, A. B., Honour, P., Borriello, S. P.: Clostridium difficile and the etiology of pseudomembranous colitis. Lancet 1, 1063-1066 (1978).

3. McDonald, L., Owings, M., Jernigan, D.: Clostridium difficile infection in patients discharged from US short-stay hospitals, 1996-2003. Emerg Inf Dis 12, 409-415 (2006).

4. Lucado, J., Gould, C., Elixhauser, A.: Clostridium difficile infections (CDI) in hospital stays. Healthcare cost and utilization project. Agency for healthcare Research and Quality. 
Statistical brief \# 124. Available at http://www.hcup-us.ahrq.gov/reports/statbriefs/sb124.pdf. Accessed on: March 27, 2012.

5. Muro, C. A., Pokrywka, M., Shutt, K., Mendelshon, A. B., Nouri, K., Posey, K., Roberts, T., Croyle, K., Krystofiak, S., Patel-Brown, S., Pasculle, A. W., Paterson, D. L., Saul, M., Harrison, L. H.: A large outbreak of Clostridium difficile-associated disease with an unexpected proportion of deaths and colectomies at a teaching hospital following increased fluoroquinolone use. Infect Control Hosp Epidmiol 26, 273-280 (2005).

6. Rholke, F., Stollman, F.: Fecal microbiota transplantation in relapsing Clostridium difficile infection. Ther Adv Gastroenterol 516, 403-420 (2012).

7. Eisemann, B., Silen, W., Bascom, G., Kauvar, A.: Fecal enema as an adjunct in the treatment of pseudomembraneous enterocolitis. Surgery 44, 854-859 (1958).

8. Borody, T. J., Warren, E.F., Leis, M. S., Surace, R., Ashman, O., Siarakas, S.: Bacteriotherapy using fecal flora. Toying with human motions. J Clin Gastroenterol 38, 475-483 (2004).

9. Debast, S. B., Bauer, M. P., Kuijper, E. J.: European Society of Clinical Microbiology and Infectious Diseases: Update of the treatment guidance document for Clostridium difficile infection. Clin Microbiol Infect 20, 1-26 (2014).

10. Surawicz, C. M., Brandt, L. J., Binion, D. G., Ananthakrishnan, A.N., Curry, S.R., Gilligan, P.H., McFarland, L.V., Mellow, M., Zuckerbraun, B. S.: Guidelines for diagnosis, treatment, and prevention of Clostridium difficile infections. Am J Gastroenterol 108, 478-498 (2013).

11. Bakken, J. S., Borody, T., Brandt, L. J., Brill, J. V., Demarco, D. C., Franzos, M. A., Kelly, C., Khoruts, A., Louie, T., Martinelli, L. P., Moore, T. A., Russell, G., Surawicz, C.: Treating Clostridium difficile infection with fecal microbiota transplantation. Clin Gastroenterol Hepatol 12, 1044-1049 (2011).

12. Gustaffson, A., Lund-Tonnensen, S., Berstad, A., Midtvedt, T., Norin, E.: Faecal shortchain fatty acids in patients with antibiotic associated diarrhea, before and after fecal enema treatment. Scand J Gastroeneterol 33, 721-727 (1998).

13. Gustaffson, A., Berstad, A., Lund-Tonnensen, S., Midtvedt, T., Norin, E.: The effect of fecal enema on five microflora associated characteristics in patients with antibiotic associated diarrhea. Scand J Gastroenterol 34, 580-586 (1999).

14. Brandt, L., Aroniadis, O., Mellow, M., Kanatzar, A., Kelly, C., Park, T., Stollman, N., Rohlke, F., Surawicz, C.: Long term follow up of colonoscopic fecal microbiota transplant for recurrent Clostridium difficile infection. Am J Gastroenterol 107, 1079-1087 (2012).

15. Mattila, E., Uusitalo-Seppala, R., Wuorela, M., Lehtola, L., Nurmi, H., Ristikankare, M., Moilanen, V., Salminen, K., Seppälä, M., Mattila, P. S., Anttila, V. J., Arkkila, P.: Fecal transplantation, through colonoscopy, is effective therapy for recurrent Clostridium difficile infection. Gastroenterology 142, 490-496 (2012).

16. Garborg, K., Waagsbo, B., Stallemo, A., Matre, J., Sundoy, A.: Results of fecal donor installation therapy for recurrent Clostridium difficile associated diarrhea. Scan J Infect Dis 42, 857-861 (2010).

17. Nieuwdorp, M., van Nood, E., Speelman, P., van Heukelem, H. A., Jansen, J. M., Visser, C. E., Kuijper, E. J., Bartelsman, J. F., Keller, J. J.: Treatment of recurrent Clostridium difficile associated diarrhea with a suspension of donor feces. Ned Tijdschr Geneeskd 152, 1927-1932 (2008). 
18. Rubin, T., Gessert, C., Aas, J.: Stool transplantation for older patients with Clostridium difficile infection. J Am Geriatr Soc 57, 2386-2387 (2009).

19. van Nood, E., Vrieze, A., Nieuwdorp, M., Fuentes, S., Zoetendal, E. G., de Vos, W. M., Visser, C. E., Kuijper, E. J., Bartelsman, J. F., Tijssen, J. G., Speelman, P., Dijkgraaf, M. G., Keller, J. J.: Duodenal infusion of donor feces for recurrent Clostridium difficile. New Eng J Med 368, 407-415 (2013).

20. Aas, J., Gessert, C. E., Bakken, J. S.: Recurrent Clostridium difficile colitis: A case series involving 18 patients treated with donor stool administered via a nasogastric tube. Clin Infect Dis 36, 580-585 (2003).

21. Cohen, S. H., Gerding, D. N., Johnson, S., Kelly, C. P., Loo, V. G., McDonald, L. C., Pepin, J., Wilcox, M. H.: Clinical practice guidelines for Clostridium difficile infection in adults: 2010 update by the society for healthcare epidemiology of America (SHEA) and the infectious diseases society of America (IDSA). Infect Control Hosp Epidemiol 31, 431-455 (2010).

22. Nagy, G. G., Várvölgyi, Cs., Balogh, Z., Orsi, P., Paragh, G.: Detailed recommendation for the methodology of fecal transplantation used for treatment of Clostridium difficile associated diarrhoea. Orv Hetil 154, 10-19 (2013). 